\title{
Características andrológicas e do sêmen de touros do composto Red Norte (Nelore $x$ Tabapuã x Red Angus x Sinepol)
}

\author{
[Andrological and semen characteristics of cross-bred Red Norte (Nelore $x$ \\ Tabapuã x Red Angus x Sinepol) young bulls] \\ R.O.D.S. Rossi, J.B. Barreto Filho ${ }^{*}$, P.H.A. Carvalho, E. Lopes, T.M. Gonçalvez \\ Universidade Federal de Lavras \\ Caixa Postal 3037 \\ 37200-000 - Lavras - MG \\ RESUMO
}

\begin{abstract}
Avaliaram-se as características andrológicas do sêmen de touros jovens do composto Red Norte (Nelore $\mathrm{x}$

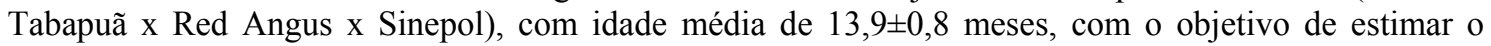
advento da puberdade e a qualidade do sêmen. Foram avaliados o perímetro escrotal (PE), o peso e as características seminais de 70 tourinhos, classificados em três grupos, de acordo com o PE: GI=27-33cm $(\mathrm{n}=24), \mathrm{GII}=33-35 \mathrm{~cm}(\mathrm{n}=24)$ e $\mathrm{GIII}=35-43 \mathrm{~cm}(\mathrm{n}=22)$. As médias de peso e a idade de cada grupo $(\mathrm{G})$ foram, respectivamente: $\mathrm{GI}=411,2 \pm 37,4 \mathrm{~kg}$ e $13,8 \pm 1,0$ meses, $\mathrm{GII}=426,9 \pm 31,5 \mathrm{~kg}$ e $14,0 \pm 0,7$ meses e $\mathrm{GIII}=438,4 \pm 38,3 \mathrm{~kg}$ e $14,0 \pm 0,6$ meses. As características seminais para cada grupo foram, volume $4,2 \pm 3,1 \mathrm{~mL}, 5,3 \pm 2,6 \mathrm{~mL}$ e $4,5 \pm 2,1 \mathrm{~mL}$; motilidade $31,3 \pm 24,1 \%, 44,2 \pm 23,9 \%$ e $43,9 \pm 21,5 \%$ e vigor $2,8 \pm 1,6$, $3,5 \pm 1,3$ e $3,5 \pm 1,3$, respectivamente. O espermiograma apresentou valores médios de concentração de $130,5 \pm 266,2 \times 10^{6} / \mathrm{mL}, 289,5 \pm 390,2 \times 10^{6} / \mathrm{mL}$ e $333,9 \pm 523,7 \times 10^{6} / \mathrm{mL}$, defeitos totais de $81,4 \pm 15,9 \%$, $73,8 \pm 15,4 \%$ e $67,9 \pm 19,0 \%$; defeitos maiores de $87,3 \pm 26,2 \%, 66,8 \pm 24,9 \%$ e $56,7 \pm 17,1 \%$ e defeitos menores de $16,6 \pm 14,9 \%, 33,2 \pm 24,9 \%$ e $43,3 \pm 17,1 \%$, respectivamente. Dos setenta animais examinados, sete $(10 \%)$ foram considerados aptos à reprodução. Os resultados mostraram que a patologia espermática diminuiu em razão do aumento do PE.
\end{abstract}

Palavras-chave: bovino, sêmen, avaliação espermática, puberdade

\begin{abstract}
Reproductive traits of cross-breed Red Norte (Nelore x Tabapuã x Red Angus x Sinepol) young bulls averaging of $13.9 \pm 0.8$ month-old were evaluated, in order to determine the puberty onset and semen quality in these animals. Scrotal circumference (SC), body weight (BW), and semen parameters of 70 bulls were measured. Animals were allotted in three groups $(G)$ according to their SC: $G I=27-33 \mathrm{~cm}$ $(n=24), G I I=33-35 \mathrm{~cm}(n=24)$, and $G I I I=35-43 \mathrm{~cm}(n=22) . B W$ and age of each group were, respectively: GI $=411.2 \pm 37.4 \mathrm{~kg}$ and $13.8 \pm 1.0$ month-old, $G I I=426.9 \pm 31.5 \mathrm{~kg}$ and $14.0 \pm 0.7$ month-old, and GIII $=438.4 \pm 38.3 \mathrm{~kg}$ and $14.0 \pm 0.6$ month-old. Seminal physical characteristics for same order of groups were: volume $4.2 \pm 3.1 \mathrm{~mL}, 5.3 \pm 2.6 \mathrm{~mL}$, and $4.5 \pm 2.1 \mathrm{~mL}$; motility $31.3 \pm 24.1 \%, 44.2 \pm 23.9 \%$, and $43.9 \pm 21.5 \%$; and vigor $2.8 \pm 1.6,3.5 \pm 1.3$, and $3.5 \pm 1.3$. The spermiogram presented concentration values of $130.5 \pm 266.2 \times 10^{6} / \mathrm{mL}, 289.5 \pm 390.2 \times 10^{6} / \mathrm{mL}$, and $333.9 \pm 523.7 \times 10^{6} / \mathrm{mL}$; total defects of $81.4 \pm 15.9 \%$, $73.8 \pm 15.4 \%$, and $67.9 \pm 19.0 \%$; major defects of $87.3 \pm 26.2 \%, 66.8 \pm 24.9 \%$ and $56.7 \pm 17.1 \%$; and minor defects of $16.6 \pm 14.9 \%, 33.2 \pm 24.9 \%$, and $43.3 \pm 17.1 \%$, for same order of groups. Seven out of 70 bulls were considered satisfactory potential breeders. Results showed that semen pathology progressively decreased when SC increased.
\end{abstract}

Keywords: bulls, semen, sperm pathology, puberty, young

Recebido em 11 de julho de 2009

Aceito em 13 de novembro de 2009

*Autor para correspondência (corresponding author)

E-mail: barreto@ufla.br 


\section{INTRODUÇÃO}

A fertilidade dos touros depende de uma série de fatores que envolvem o comportamento e as condições físicas do animal, além daqueles diretamente correlacionados com o sêmen, como a motilidade espermática, níveis específicos de determinadas proteínas de membrana e composição bioquímica do plasma seminal (Assumpção et al., 2005). Diversas mensurações também devem ser levadas em consideração, quando se pretende avaliar o potencial de fertilidade de um touro. O perímetro escrotal (PE), como medida indireta da massa testicular e a forma, o comprimento e a largura dos testículos são dimensões auxiliares (Abdel-Razek e Ali, 2005).

A avaliação andrológica dos touros, realizada antes da aquisição do animal ou do uso, tem o objetivo de identificar as condições patológicas e a estrutura dos testículos e das glândulas acessórias (McGowan et al., 2002). Essas avaliações permitem a identificação do estádio de maturidade sexual e a fertilidade futura, baseada no desenvolvimento de parâmetros mensuráveis, em animais jovens. Segundo Jimenez-Severiano (2002), existe correlação positiva entre o desenvolvimento do trato reprodutivo de touros e a dimensão do $\mathrm{PE}$, fato já verificado por outros autores (MacMillan e Hafs, 1969; Amann, 1983). Alguns trabalhos (Kastelic et al., 2001; Brito et al., 2002) demonstraram que as dimensões testiculares e do escroto podem ser utilizadas para predizer a produção espermática e a qualidade seminal.

Segundo Wolf et al. (1965), a puberdade do touro é definida quando o sêmen do animal apresentar concentração $\geq 50 \times 10^{6} \mathrm{de}$ espermatozoides/ejaculado e motilidade $\geq 10 \%$. A maturidade sexual ocorre quando houver dois ejaculados consecutivos contendo $\geq 500 \times 10^{6}$ espermatozoides/ejaculado e pelo menos um dos ejaculados exibir motilidade $\geq 50 \%$, ou quatro ejaculados sucessivos com $\geq 500 \times 10^{6}$ espermatozoides (Tatman et al., 2004).

Os objetivos deste trabalho foram investigar as características seminais e a biometria testicular de touros jovens do composto Red Norte (Nelore x Tabapuã x Red Angus x Sinepol) e avaliar o estádio de desenvolvimento sexual desses animais.

\section{MATERIAL E MÉTODOS}

Foram utilizados 70 tourinhos do composto Red Norte (Nelore x Tabapuã x Red Angus x Sinepol) em fase final de prova de ganho de peso, classificados em três grupos $(\mathrm{G})$, de acordo com o PE: GI $=27-33 \mathrm{~cm}(\mathrm{n}=24), \mathrm{GII}=33-35 \mathrm{~cm}$ $(n=24)$ e $G I I I=35-43 \mathrm{~cm}(n=22)$. Para a colheita de sêmen, foi utilizada a eletroejaculação ou a massagem das ampolas dos ductos deferentes.

Avaliaram-se o PE, o volume do ejaculado e os parâmetros físicos do sêmen, turbilhonamento, motilidade e vigor. Duas alíquotas do sêmen de cada animal foram retiradas e armazenadas em solução de formol-salina tamponada, para posterior avaliação da concentração e patologia espermáticas, usando-se microscopia de luz e de contraste de fase, respectivamente. Os defeitos espermáticos foram classificados de acordo com Barth e Oko (1989), e os critérios adotados para determinação da puberdade e maturidade sexual foram os de Wolf et al. (1965) e Tatman at al. (2004), respectivamente.

A análise estatística foi realizada pelo programa SISVAR (Ferreira, 2000), comparando-se as médias pelo teste Scott-Knott, com nível de significância de $5 \%$.

\section{RESULTADOS E DISCUSSÃO}

Sete animais $(10 \%)$ foram considerados aptos à reprodução, seguindo os critérios propostos pelo Colégio Brasileiro de Reprodução Animal (Manual..., 1998). Em relação ao PE, os animais foram classificados como: GI, questionável a bom; GII, bom e GIII, muito bom, de acordo com as diretrizes da Beef Improvement Federation (Beef..., 1996) (Tab. 1). Os valores médios de $\mathrm{PE}$ foram diferentes $(\mathrm{P}<0,05)$ entre os grupos, e o peso vivo foi diferente $(\mathrm{P}<0,05)$ entre o GI e GIII. A idade entre esses grupos não diferiu estatisticamente. Observou-se correlação positiva $(\mathrm{r}=0,29 ; \mathrm{P}<0,05)$ entre $\mathrm{PE}$ e peso vivo. Em estudo com touros da raça Nelore, Moura et al. (2002) também verificaram correlação semelhante, e Freneau et al. (2006) também verificaram aumento do $\mathrm{PE}$ em animais mais pesados. 
Tabela 1. Peso vivo, idade e perímetro escrotal nos diferentes grupos de tourinhos do composto Red Norte (Nelore x Tabapuã x Red Angus x Sinepol) em regime de confinamento

\begin{tabular}{lccc} 
& GI $(\mathrm{n}=24)$ & GII $(\mathrm{n}=24)$ & GIII $(\mathrm{n}=22)$ \\
\hline Peso vivo (kg) & $411,2 \pm 37,4 \mathrm{~b}$ & $426,9 \pm 31,5 \mathrm{ab}$ & $438,4 \pm 38,3 \mathrm{a}$ \\
Idade (meses) & $13,8 \pm 1,0$ & $14,0 \pm 0,7$ & $14,0 \pm 0,6$ \\
$\mathrm{PE}(\mathrm{cm})$ & $31,3 \pm 1,3 \mathrm{c}$ & $34,6 \pm 0,8 \mathrm{~b}$ & $37,1 \pm 1,6 \mathrm{a}$ \\
\hline
\end{tabular}

Letras distintas na mesma linha indicam diferença estatística entre os valores pelo teste Scott-Knott a 5\%.

Em estudo com touros de corte com idade média de 16 meses, Kastelic et al. (2001) encontraram PE médio de $33,7 \pm 0,3 \mathrm{~cm}$, com variação de 27 a $41 \mathrm{~cm}$. Arteaga et al. (2001), em trabalho com touros jovens entre 11 e 15 meses, verificaram $\mathrm{PE}$ de 33,4 a $35,3 \mathrm{~cm}$. Sabe-se que o crescimento mais intenso dos testículos ocorre próximo à puberdade (Dal-Farra et al., 1998), e isso também foi observado nos tourinhos deste trabalho. Contudo, a qualidade do sêmen de $90 \%$ dos animais ainda apresentava características de imaturidade sexual.

Considerando que o aparecimento de espermatozoides no ejaculado é indicador de precocidade sexual, os animais deste experimento com mais de $13,8 \pm 1,0$ meses e $31,3 \pm 1,3 \mathrm{~cm}$ de perímetro escrotal já estavam aptos a produzir espermatozoides. Observou-se correlação positiva $(\mathrm{r}=0,24 ; \mathrm{P}<0,05)$ entre concentração espermática e PE. Segundo Unaniam (1997), tourinhos da raça Nelore com média de idade de 13,6 meses e $22,9 \mathrm{~cm}$ de $\mathrm{PE}$ apresentavam espermatozoides no ejaculado.

As características físicas do ejaculado (Tab. 2) não diferiram $(\mathrm{P}>0,05)$ entre os grupos estudados. $\mathrm{O}$ volume médio encontrado foi de $4,6 \pm 2,7 \mathrm{~mL}$ para a idade média de $13,9 \pm 0,8$ meses. Estudos com animais da raça Nelore (Freneau et al., 2006) demonstraram que aos 14 meses o volume foi de $3,1 \pm 0,4 \mathrm{~mL}$, e em animais da raça Gir (Martinez et al., 2000), nessa mesma idade, de $5,16 \pm 1,73 \mathrm{~mL}$. Os resultados deste estudo mostraram que em relação ao volume de sêmen, os tourinhos apresentaram características de puberdade.

Tabela 2. Características físicas dos ejaculados de tourinhos do composto Red Norte (Nelore x Tabapuã x

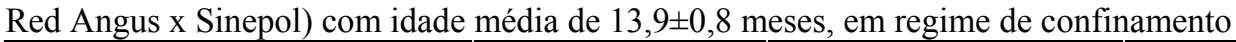

\begin{tabular}{lccc}
\hline & GI $(\mathrm{n}=24)$ & GII $(\mathrm{n}=24)$ & GIII $(\mathrm{n}=22)$ \\
\hline Volume (mL) & $4,2 \pm 3,1$ & $5,3 \pm 2,6$ & $4,5 \pm 2,1$ \\
Motilidade (\%) & $31,3 \pm 24,1$ & $44,2 \pm 23,9$ & $43,9 \pm 21,5$ \\
Vigor (0-5) & $2,8 \pm 1,6$ & $3,5 \pm 1,3$ & $3,5 \pm 1,3$ \\
Concentração (x106/mL) & $130,5 \pm 266,2$ & $289,5 \pm 390,2$ & $333,9 \pm 523,7$ \\
Defeitos totais (\%) & $81,4 \pm 15,9 \mathrm{a}$ & $73,8 \pm 15,4 \mathrm{ab}$ & $67,9 \pm 19,0 \mathrm{~b}$ \\
Defeitos maiores (\%) & $87,3 \pm 26,2 \mathrm{a}$ & $66,8 \pm 24,9 \mathrm{~b}$ & $56,7 \pm 17,1 \mathrm{~b}$ \\
Defeitos menores (\%) & $16,6 \pm 14,9 \mathrm{~b}$ & $33,2 \pm 24,9 \mathrm{a}$ & $43,3 \pm 17,1^{\mathrm{a}}$ \\
\hline
\end{tabular}

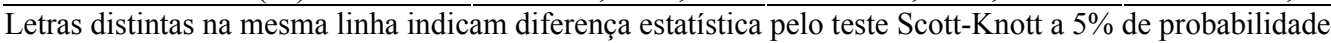

Em trabalho realizado com touros cruzados e Nelore, Valentim et al. (2002) mostraram o maior potencial de produção espermática dos mestiços $(\mathrm{PE}=29,8 \mathrm{~cm}, 20$ meses de idade $)$ quando comparados aos zebuínos $(\mathrm{PE}=27,4 \mathrm{~cm}$, 20 meses de idade). Tomando-se o crescimento do PE como critério de avaliação da maturidade sexual, segundo esses autores, os touros cruzados amadureceram mais cedo que os zebuínos.

A avaliação da cinética espermática resultou nos valores médios de $39,6 \pm 23,7 \%$ e $3,3 \pm 1,4$ para motilidade individual e vigor, respectivamente. Estes valores ficaram abaixo dos encontrados por Assumpção et al. (2005) em touros Nelore, 60$90 \%$ e 3-5, respectivamente, e por Martinez et al. (2000), em animais da raça Gir, 59,3\% e 4,9, respectivamente. A concentração espermática média foi de $250,7 \times 10^{6}$ espermatozoides $/ \mathrm{mL}$. A concentração ficou abaixo dos valores encontrados nas raças Gir (Martinez et al., 2000) e Nelore (Assumpção et al., 2005), e de touros jovens de origem européia (Arteaga et al., 2001), possivelmente em razão do método de coleta e 
não condicionamento dos animais à eletroejaculação. Segundo com os critérios de Wolf et al. (1965), todos os animais do presente estudo seriam classificados como púberes, e apenas dois animais do GII e do GIII seriam considerados maduros sexualmente, de acordo com os critérios de Tatman et al. (2004).

A avaliação da morfologia espermática (Tab. 2) revelou que os animais do GI apresentaram maior patologia em relação aos do GIII $(\mathrm{P}<0,05)$. Segundo o Colégio Brasileiro de Reprodução Animal (Manual..., 1998), o total de espermatozoides anormais não deve ultrapassar a $30 \%$ para que o reprodutor seja considerado apto à reprodução. $\mathrm{O}$ total de patologia variou de $28 \mathrm{a}$ $100 \%$, com média de $74,5 \%$. Kastelic et al. (2001) estudaram 129 touros de corte e encontraram $62,1 \pm 1,5 \%$ de células espermáticas normais e variação de 13 a 97\%; 7,3 $\pm 0,6 \%$ de defeitos maiores e variação de 0 a $45 \%$; e $30,6 \pm 1,4 \%$ de defeitos menores e variação de 2 a $77 \%$. Neste estudo, os defeitos encontrados foram a gota citoplasmática proximal e a patologia da peça intermediária, semelhante aos resultados encontrados por Arteaga et al. (2001), em touros considerados imaturos sexualmente.

Neste experimento, os animais foram submetidos a uma dieta com $80 \%$ de concentrado e $20 \%$ de volumoso, durante o período da prova de ganho de peso, fato que pode ter acarretado efeito deletério na qualidade seminal, principalmente no que diz respeito à morfologia espermática. $\mathrm{O}$ prejuízo à qualidade seminal em animais que recebem dietas com alto teor energético já foi mencionado por Coulter et al. (1997).

\section{CONCLUSÕES}

Em função da avaliação de características seminais e desenvolvimento testicular, os tourinhos examinados encontravam-se em fase de transição do estádio de imaturidade sexual para a puberdade. Considerando-se os mesmos critérios para avaliação de aptidão reprodutiva, $10 \%$ dos animais foram considerados maduros sexualmente e aptos à reprodução. Estes dados reiteram a importância da avaliação andrológica e da utilização do PE como critério de seleção para precocidade sexual em bovinos, em programas de seleção e melhoramento genético.

\section{REFERÊNCIAS BIBLIOGRÁFICAS}

ABDEL-RAZEK, A.K.; ALI, A. Developmental Changes of Bull (Bos taurus) Genitalia as Evaluated by Caliper and Ultrasonography. Reprod. Dom. Anim., v.40, p.23-27, 2005.

AMANN, R.P. Endocrine changes associated with onset of spermatogenesis in Holstein bulls. J. Dairy Sci., v.66, p.2606-2622, 1983.

ARTEAGA, A.; BARACALDO, M.; BARTH, A.D. The proportion of beef bulls in western Canada with mature spermiograms at 11 to 15 months of age. Can. Vet. J., v.42, p.783-787, 2001.

ASSUMPÇÃO, T.I.; TORRES JÚNIOR, R.A.A.; SOUSA, M.V. et al. Correlation between fertility and levels of protein, sugar and free amino acids in seminal plasma of Nelore bulls. Arq. Bras. Med. Vet. Zootec., v.57, p.5561, 2005.

BARTH, A.D.; OKO, R.J. Abnormal morphology of bovine spermatozoa. Ames: Iowa State University Press, 1989.

BEEF IMPROVEMENT FEDERATION. Guidelines for uniform beef improvement programs. Raleigh: U.S. Department of Agriculture, North Carolina State University, 1996. 155p.

BRITO, L.F.; SILVA, A.E.; RODRIGUES, L.H. et al. Effect of age and genetic group on characteristics of scrotum, testes and testicular vascular cones and on sperm production and semen quality in AI bulls in Brazil. Theriogenology, v.58, p.1175-1186, 2002.

COULTER, G.H.; COOK, R.B.; KASTELIC, J.P. Effects of dietary energy on scrotal surface temperature, seminal quality, and sperm production in young beef bulls. J. Anim. Sci., v.75, p.1048-1052, 1997.

DAL-FARRA, R.A.; LOBATO, J.F.; FRIES, L.A. Fatores de correção do perímetro escrotal para efeitos de idade e peso ao sobreano de tourinhos Nelore. Rev. Bras. Zootec., v.27, p.1092-1096, 1998. 
FERREIRA, D.F. Análises estatísticas por meio do SISVAR (Sistema para Análise de Variância) para Windows ${ }^{\circledR}$ versão 4.0. In: REUNIÃO ANUAL DA REGIÃO BRASILEIRA DA SOCIEDADE INTERNACIONAL DE BIOMETRIA, 45., 2000, São Carlos. Anais... São Carlos: UFSCar, 2000. p.255-8.

FRENEAU, G.E.; VALE FILHO, V.R.; MARQUES Jr., A.P. et al. Puberdade em touros Nelore criados em pasto no Brasil: características corporais, testiculares e seminais e de índice de capacidade andrológica por pontos. Arq. Bras. Med. Vet. Zootec., v.58, p.1107-1115, 2006.

JIMENEZ-SEVERIANO, H. Sexual development of dairy bulls in the Mexican tropics. Theriogenology, v.58, p.921-932, 2002.

KASTELIC, J.P.; COOK, R.B.; PIERSON, R.A. et al. Relationships among scrotal and testicular characteristics, sperm production, and seminal quality in 129 beef bulls. Can. J. Vet. Res., v.65, p.111-115, 2001.

MacMILLAN, K.L.; HAFS, H.D. Reproductive tract of Holstein bulls from birth through puberty. J. Anim. Sci., v.28, p.233-239, 1969.

MANUAL para exame andrológico e avaliação de sêmen animal. 2.ed. Belo Horizonte: Colégio Brasileiro de Reprodução Animal, 1998.

MARTINEZ, M.L.; VERNEQUE, R.S.; TEODORO, R.L. et al. Correlações entre características da qualidade do sêmen e a circunferência escrotal de reprodutores da raça Gir. Rev. Bras. Zootec., v.29, p.700-706, 2000.
McGOWAN, M.R.; BERTRAM, J.D.; FORDYCE, G. et al. Bull selection and use in northern Australia. 1. Physical traits. Anim. Reprod. Sci., v.71, p.25-37, 2002.

MOURA, A.A.A.; RODRIGUES, G.C.; MARTINS FILHO, R. Desenvolvimento ponderal e testicular, concentrações periféricas de testosterona e características de abate em touros da raça Nelore. Rev. Bras. Zootec., v.31, p.934-943, 2002.

TATMAN, S.R.; NEUENDORFF, D.A.; WILSON, T.W. et al. Influence of season of birth on growth and reproductive development of Brahman bulls. Theriogenology, v.62, p.93-102, 2004.

UNANIAM, M.M. A procura de marcadores de precocidade em gado Nelore. In: O NELORE DO SÉCULO XXI. NELORE PRECOCE: SELEÇÃO, PRODUÇÃO E COMERCIALIZAÇÃO, 4, 1997, Uberaba. Anais...Uberaba: ABCZ-ABCN, 1997. p.51-57.

VALENTIM, R.; ARRUDA, R.P.; ALENCAR, M.M. et al. Biometria testicular de touros Nelore (Bos taurus indicus) e touros cruzados Neloreeuropeu (Bos taurus indicus x Bos taurus taurus) aos 20 e 24 meses de idade. Braz. J. Vet. Res. Anim. Sci., v.39, p.113-120, 2002.

WOLF, F.R.; ALMQUIST, J.O; HALE, E.B. Prepuberal behavior and puberal characteristics of beef bulls on high nutrient allowance. J. Anim. Sci., v.24, p.761-765, 1965. 